\title{
IMPACT AND CRASHWORTHINESS CHARACTERISTICS OF VENERA TYPE LANDERS FOR FUTURE VENUS MISSIONS
}

\author{
Kevin Schroeder ${ }^{(1)}$ and Javid Bayandor ${ }^{(1)}$ \\ And
}

Jamshid Samareh $^{(2)}$

In this paper an in-depth investigation of the structural design of the Venera 9-14 landers is explored. A complete reverse engineering of the Venera lander was required. The lander was broken down into its fundamental components and analyzed. This provided insights into the hidden features of the design. A trade study was performed to find the sensitivity of the lander's overall mass to the variation of several key parameters. For the lander's legs, the location, length, configuration, and number are all parameterized. The size of the impact ring, the radius of the drag plate, and other design features are also parameterized, and all of these features were correlated to the change of mass of the lander.

A multi-fidelity design tool used for further investigation of the parameterized lander was developed. As a design was passed down from one level to the next, the fidelity, complexity, accuracy, and run time of the model increased. The low-fidelity model was a highly nonlinear analytical model developed to rapidly predict the mass of each design. The medium and high fidelity models utilized an explicit finite element framework to investigate the performance of various landers upon impact with the surface under a range of landing conditions.

This methodology allowed for a large variety of designs to be investigated by the analytical model, which identified designs with the optimum structural mass to payload ratio. As promising designs emerged, investigations in the following higher fidelity models were focused on establishing their reliability and crashworthiness. The developed design tool efficiently modelled and tested the best concepts for any scenario based on critical Venusian mission requirements and constraints. Through this program, the strengths and weaknesses inherent in the Venera-Type landers were thoroughly investigated. Key features identified for the design of robust landers will be used as foundations for the development of the next generation of landers for future exploration missions to Venus.

\section{Nomenclature}

$=\operatorname{Area}\left(m^{2}\right)$

$=$ Young's modulus $(\mathrm{Pa})$

$=$ Weight $(N)$

$=$ Factor of safety

$=$ Internal energy $(J)$

$=\operatorname{Mass}(\mathrm{kg})$

$=$ External pressure $(\mathrm{Pa})$

$=$ Pressure vessel radius $(m)$

$=$ Honeycomb radius in each leg $(m)$

$=$ Stroke $(m)$

$=$ Thickness of the pressure vessel $(m)$

$=$ Velocity $(\mathrm{m} / \mathrm{s})$

$=$ Leg angle from vertical (deg)

$=$ Energy absorber crush strength $(\mathrm{Pa})$

${ }^{I}$ Crashworthiness for Aerospace Structure and Hybrids (CRASH) Lab, Virginia Tech Blacksburg, VA, 24061 USA, Bayandor@vt.edu

${ }^{2}$ NASA Langley Research Center, Hampton, VA, United States 


\section{BACKGROUND}

A number of Soviet probes made it through the thick cloud layer to find the extremely harsh conditions beneath. The Venusian atmosphere is considerably thicker than that of Earth, but the pressure and temperatures above its dense cloud layer are relatively benign. Beneath the cloud layer the temperatures and pressures begin to spike. In the last $100 \mathrm{~km}$ to the surface the temperatures jump from approximately $100 \mathrm{~K}$ to $750 \mathrm{~K}$. At the surface the atmospheric pressure is approximately 92 bars.

Despite these significant challenges, the Soviet's Venus campaign, Venera, continued to send probes to Venus ${ }^{1}$ (see Figure 1). In 1970 the Venera 7 became the first spacecraft to successfully land on the surface of Venus $^{2}$. A number of improvements were made for the next mission, Venera 8 , and by the time the Venera 9 was launched and entirely new lander had been designed ${ }^{3}$. Unlike the previous probes from the Venera campaign which were focused on analyzing the atmospheric properties as they descended, the new Venera lander was designed to perform various additional scientific experiment on the surface.

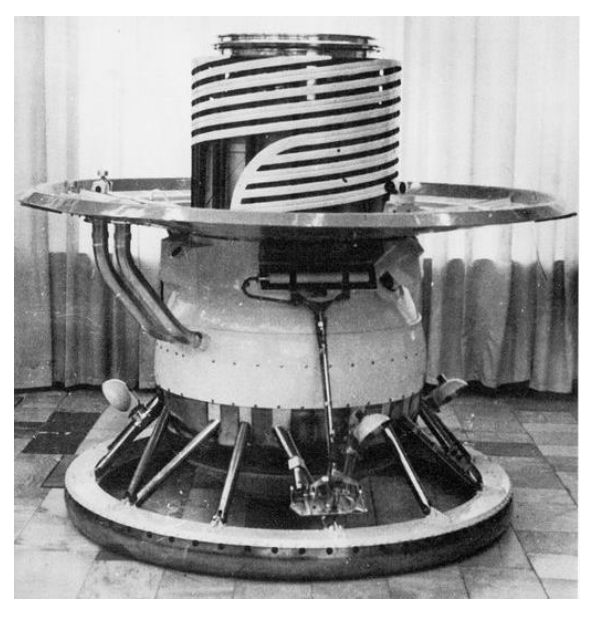

Figure 1: Venera 9 Lander $^{1}$

The lander entered the atmosphere at $10.7 \mathrm{~km} / \mathrm{s}$, encapsulated in a $2.4 \mathrm{~m}$ spherical heat shield. After a series of parachutes, the lander was extracted from its heat shield and its speed was reduced to below $50 \mathrm{~m} / \mathrm{s}$. At this point, the lander and its payload became exposed to ambient condition and although the temperature at this altitude is several hundred degrees less that the temperature at the surface, it was important to get the lander to the surface quickly before the payload was compromised by the high temperatures. One last three-domed parachute was deployed to slow the descent through the cloud sheet for atmospheric readings but was released as soon as the lander had fully passed through cloud layer. Because there is a significant increase of the atmospheric density in the last $50 \mathrm{~km}$ to the surface, the speed of the lander was able to quickly decelerate in free fall to approximately $7 \mathrm{~m} / \mathrm{s}^{4}$.

The primary structures of the Venera lander were analyzed as four distinct components. From top down, those four component are the parachute capsule and drag plate, the payload module, the legs, and the impact ring. For simplicity's sake, the parachute capsule and the drag plate are grouped into one component because although both components are necessary for descent, they do not add much to the structural response of the lander at impact. The payload module was comprised of a pressure vessel, an internal stiffener, the payload contained within it, and the insulation surrounding it. The payload module was roughly one meter in diameter, $80 \mathrm{~cm}$ diameter pressure vessel with the remaining thickness contributed to by the insulation ${ }^{3}$. All the atmospheric sensitive payload was housed inside the spherical pressure vessel 
with the insulation surrounding them both. Although the payload of each mission is known to have changed, the overall size and design remained relatively constant.

Below the payload module is what many schematics label "Посадочное устройство" which directly translates as "landing gear." In this paper, the landing gear was broken into two components: the legs and the impact ring. Unfortunately, most resources do not go into any additional detail other than the name. Of the sources that do include more information on the landing gear, some sources seem to contradict others. There is a consensus in the literature that the impact ring was a hollow metallic torus, which would crush on impact ${ }^{2,3,4,5}$ but both Bond and Soediono mention that impact ring was attached to some type of shock absorber. Presumably, Bond and Soediono where saying that legs were some type of shock absorber, while Ball, Keldysh, and many more make no mention of the legs. To add to the ambiguity, none of the publically available schematics of the Venera and Vega landers show any internal components of the legs. Based on the available literature, the original design of the Venera lander legs is inconclusive. While this information may be accessible to some, it is either classified or somehow lost in translation.

In light of this, much of the effort invested in this work was dedicated to defining a new design of the landing gear for a baseline of the Venera-type landers. Despite the gap in the literature with regards to the legs, there is a fair amount of information that was helpful in designing a baseline. There were 18 legs that connected the payload module to the impact ring below. Due to their short length and small diameter it is likely that they were either rigid or a secondary energy absorbers used to isolate payload from much of the shock wave due to impact. The legs also provided the added benefit of keeping the payload module off the ground. Because a wide scale survey of Venus wasn't preformed until NASA's Pioneer Venus Mission in 1978, the topography of the surface was largely unknown ${ }^{6}$. Even today due to the thick cloud layer the resolution of the mapped surface is relatively coarse. If the lander happened to impact on a very rocky terrain, the payload module's pressure vessel would be susceptible to a penetration. Due to the extremely high pressure at the surface, a perturbation to the pressure vessel could lead buckling of the pressure vessel which could damage or destroy the payload.

The impact ring is a hollow metallic ring, which would crush on impact converting much of the kinetic energy of the lander into plastic strain. Additionally, the impact ring had holes perforating the outer surface of the metallic shell. These holes, seen in most images of the lander, had several interesting characteristics. The obvious reason for these holes is to equalize the atmospheric pressure pushing on the outside of the ring with the internal pressure. But because of these holes allowed the ring to be filled with the dense atmospheric gases when the impact ring was crushed, the high density fluid forced out of these holes added some viscous damping to the impact sequence . 
Overall, this design proved to be extremely robust and reliable. Its basic design was used for all the remaining missions to the surface of Venus. While the new baseline Venera-type lander will not be identical to the original design an effort was made to keep the design similar to ensure that the baseline will be as robust and reliable.

\section{ENERGY ABSORBERS}

Energy absorbers can be classified by the mechanism used to dissipate or absorb the energy. Classical energy absorbers use friction and damping as well as irreversible deformation ${ }^{7}$. The Venera lander likely utilized both classifications. Although both method were viable options, each one presents a significant complication.

Because neither Bond nor Soediono ${ }^{2}$ specified a specific type of shock absorber, a verity of options were considered for the new baseline model. Viscous dampers are a possibility that could have been used in the original Venera lander, although a number of obstacles must have been overcome. The design of such a mechanism will need to overcome the effects of extreme temperature change. Within approximately one hour the fluid will transition from the temperature maintained in transit to the planet to the surface temperature, approximately $485^{\circ} \mathrm{C}$. Under this variation most fluids will be subject to phase change, as well as change in specific volume, viscosity, and several other critical properties. The variation of fluid with temperature will make it a challenge to design the dampers valve accurately.

A better energy absorption option for Venera lander is irreversible deformation using landing legs: the legs will utilize plastic deformation/buckling of the legs as a form of shock absorption. This could potentially explain the absence of an internal structure in any of the schematics. This mechanism would be significantly simpler to account for the temperature change. However deforming the legs was likely not the method chosen as it loses the added benefit of keeping the payload module off the ground. Using this mechanism would lead to a decrease in reliability of the lander and would increase the risk of penetrating the pressure vessel.

The hybrid mechanism used in the impact ring was a clever utilization the landers environment. While the original impact ring is extremely mass effective the mechanism is complicated to predict analytically and computationally. This is not conducive for rapidly analyzing various designs and thus may not be suitable for the baseline model.

Because of these complications none of the aforementioned energy absorbers were chosen for the new baseline design. Alternatively, the use of crushable honeycomb and metallic foams was substituted in place of those mechanisms to provide lightweight, highly predictable impact characteristics. Metallic honeycomb was not used for the Venera lander because although its first use as an energy absorber for the Apollo 11 landing gear predated the design of the Venera lander by a couple of years, the technology was still extremely new and was not yet a commonly used energy absorber ${ }^{8}$. 
As far as compressive energy-absorbers are concerned, honeycomb and metallic foams offer near ideal energy absorption characteristics. The energy absorption can be broken into three phases: initiation, plastic deformation, and densification, as seen in Figure 2. The initiation phase occurs on impact and is characterized by purely elastic deformation. That is, if the deformation does not exceed the initiation phase, the material will return back to a zero stress and zero strain state. However, after the initiation phase is surpassed, the thin walls of the honeycomb structure will begin to buckle in a near uniform periodic distance. This progressive buckling behavior is what contributes to the near ideal energy absorbing characteristics of honeycomb. Throughout the plastic deformation phase, impact energy is absorbed as a constant rate until the densification phase is reached. When the material is fully condensed the stress/strain relationship returns to a near linear state. Honeycomb materials can crush up to $80 \%$ strain before the densification phase begins.

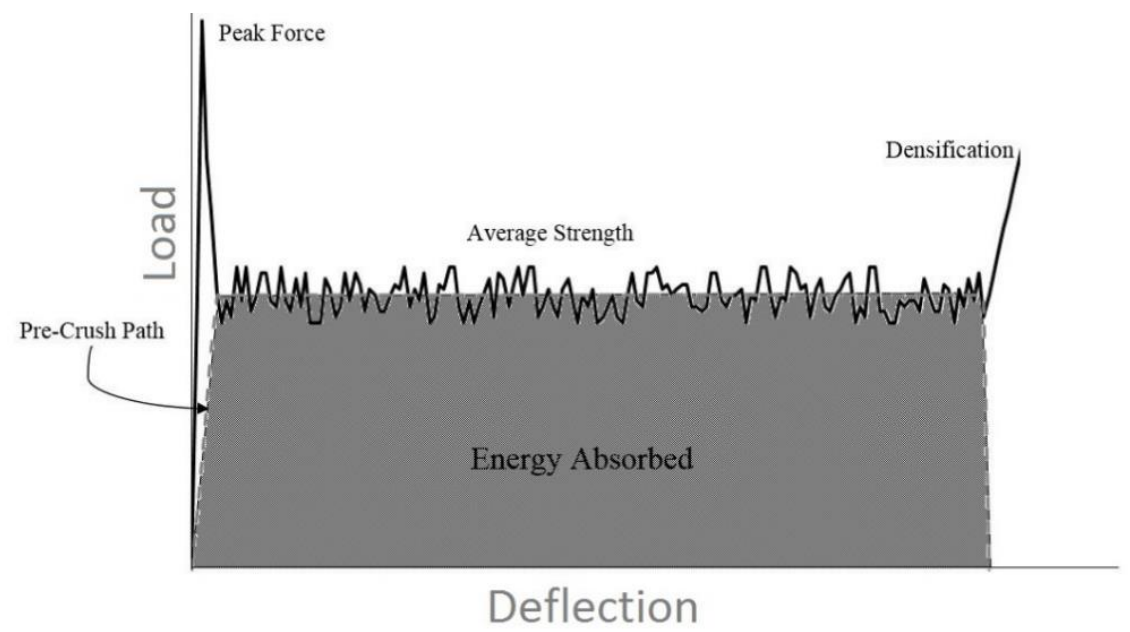

Figure 2: Honeycomb Load-Deflection Plot

The average crush load of the honeycomb can be tailored by the geometry of the honeycomb cell and the thickness of the cell wall. Thus, a wide range of crush strengths can be attained by simply adjusting the geometry or material of the honeycomb. Better impact characteristics for honeycomb can be obtained from a procedure called precrushing. Pre-crushing applies a load to the honeycomb and allows the material to surpass the peak force start buckling before it is unloaded. Now that the buckling has been initiated, the peak load can be bypassed the next time the material is loaded.

Metallic foams respond similar to honeycomb but with some differences. Metallic foam typically reach densification before honeycombs at roughly $60 \%$ strain. Thus foams have a smaller specific energy absorption (SEA) than honeycomb, however, they are effectively an isotropic material. While honeycomb is restricted to a specific orientation and in some cases requires a guide, metallic foams can be crushed predictably under any impact orientation. 
For these reasons, the investigation of the Venera-type landers for future missions to Venus will utilize honeycomb legs and a low density open cell metallic foam for the impact ring. Honeycomb legs concept is a reasonable choice for many of the reasons mentions above in addition to the fact that the legs are already designed to only apply an axial load. This will facilitate the proper guided crushing of the honeycomb without adding any complexity. The impact ring on the other hand, will be better suited by the metallic foam because it is likely that the ring will not impact perfectly normal to the surface.

\section{MULTI-FIDELITY DESIGN TOOL}

In order to see how the design of each component affects the lander as a whole, it was important to analyze each component individually. With the vast number of different combinations, it was impractical to model and test all the different combinations. Instead, system was created to systematically analyze the multiple variations at three distinct levels of fidelity.

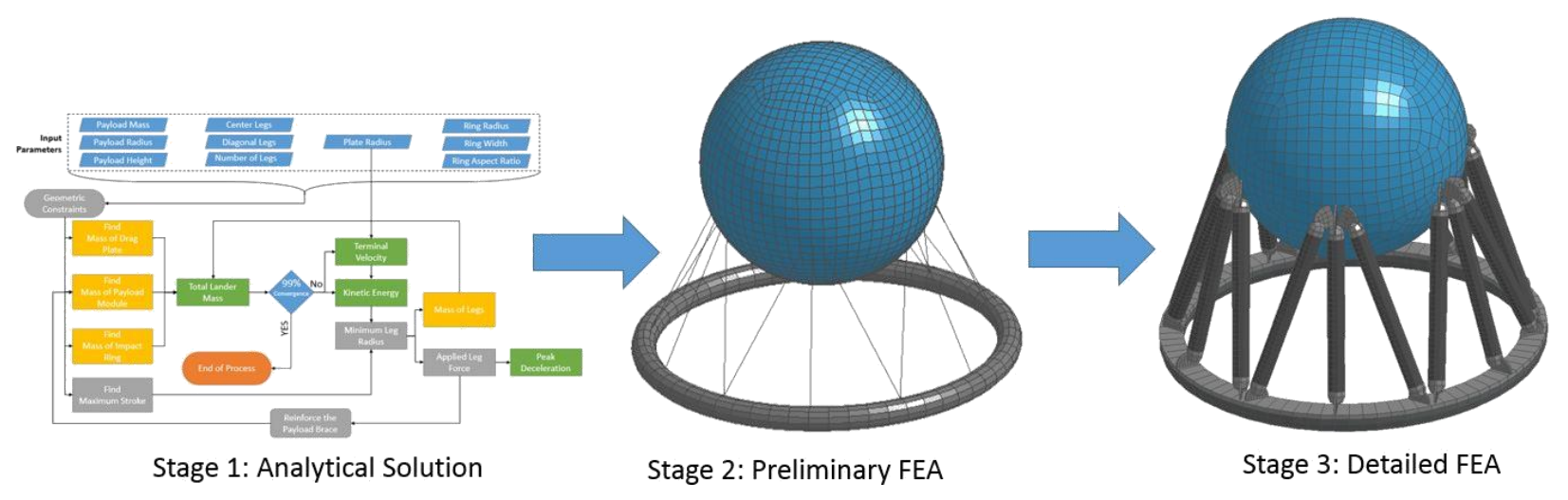

Figure 3: Overview of Multi-Fidelity Design Tool

Figure 3 gives a brief overview of different levels of fidelity that were created. The low fidelity model makes an analytical approximation of the impact response of the lander. This model is able to provide highly reliable predictions of the lander's total mass, impact velocity, and peak deceleration at impact for an ideal impact. The medium fidelity model automatically creates a finite element model based on imported data from the low fidelity model. This model is used to test various impact scenarios at a low computational expense. The high fidelity model offers a more detailed simulation than the previous model but is more time-expensive, both to build and to run. However, the high fidelity model is able to accurately query the stress concentrations in the model. It is noteworthy to mention that neither the medium nor high fidelity models explicitly model the drag plate and parachute capsule because they offer minimal structural contributions at impact. However, both levels include its effect on the landers impact speed and total mass. 
From this multi-fidelity design tool, the characteristics inherent in the design of the Venera lander can be investigated efficiently. The low-fidelity model can rapidly analysis dozens of variations within seconds. From there data from the most promising designs can be passed up to the medium-fidelity to dynamically simulate the various impact conditions with a still relatively low run time. When a final design is chosen, its parameter are used to create the highfidelity model to investigate the detail of the model.

\section{A. Low-Fidelity - Analytical Model}

Each of the four key components mentioned above were approximated as simple geometric shape so that the volume of material that comprised them and thus their mass could be calculated. Most of the major components could be modeled as classical shapes; the payload module as a hollow sphere with uniform thickness, the legs as cylinders, and the impact ring as a torus. But the drag plate and some of the minor components (e.g., the joints connecting the legs to the impact ring and payload, and a stiffening ring located inside the pressure vessel) had to be modeled in an alternative manners. From this work, a reasonably accurate account of how each component attributed to the landers overall mass was made.

An additional benefit to modeling each component analytically is that the geometry could easily be parameterized and modified to predict how the lander will behave for a baseline impact scenario. Each major component could be varied with respect to the others to see how it would affect the impact characteristics of the lander as a whole. These results together with the estimated mass of each lander configuration would be used to find the most preferable lander designs.

Within the design of the Venera lander, there are dozens major parameters that can be varied. In order to analytically predict the mass and impact characteristics of any lander configuration, a systematic approach need to be employed to simplify the process. For this reason, many of the design parameters were found to be dependent on some other parameter. For example, the amount of honeycomb needed to properly absorb the kinetic energy can be calculated from the landers kinetic energy, which is a function of the landers mass and terminal velocity. Additionally, the required thickness of the payload module to prevent buckling under hydrostatic pressure is a function of the pressure vessels radius. By finding multiple relationships such as these and keeping some of the obviously minor variables fixed, the entire model was simplified down to 10 major design variables. The payload module was sized as a function of its radius and mass. The distance the payload module initially was above the impact ring was modeled by the parameter Payload Height. The impact ring was modeled based on its radius, width, and the metallic foam's aspect ratio that controlled the 
foams thickness. The drag plate was sized solely as a function of its radius. And finally, the legs were modeled based on the number of legs and configuration (two parameters).

A process overview of the low-fidelity model is shown in Figure 4. The 10 input parameters, highlighted blue in Figure 4, would be used to size the drag plate, the payload module, and the impact ring. Together, those masses would be used as the initial estimate of the landers total mass. The total mass was used to calculate the terminal velocity and the kinetic energy of the lander, which in turn was used to size the legs. The additional mass of the leg was then fed back into the total mass of the lander for the next iteration. The iterative solver continued until a $99 \%$ convergence of the lander total mass was achieved.

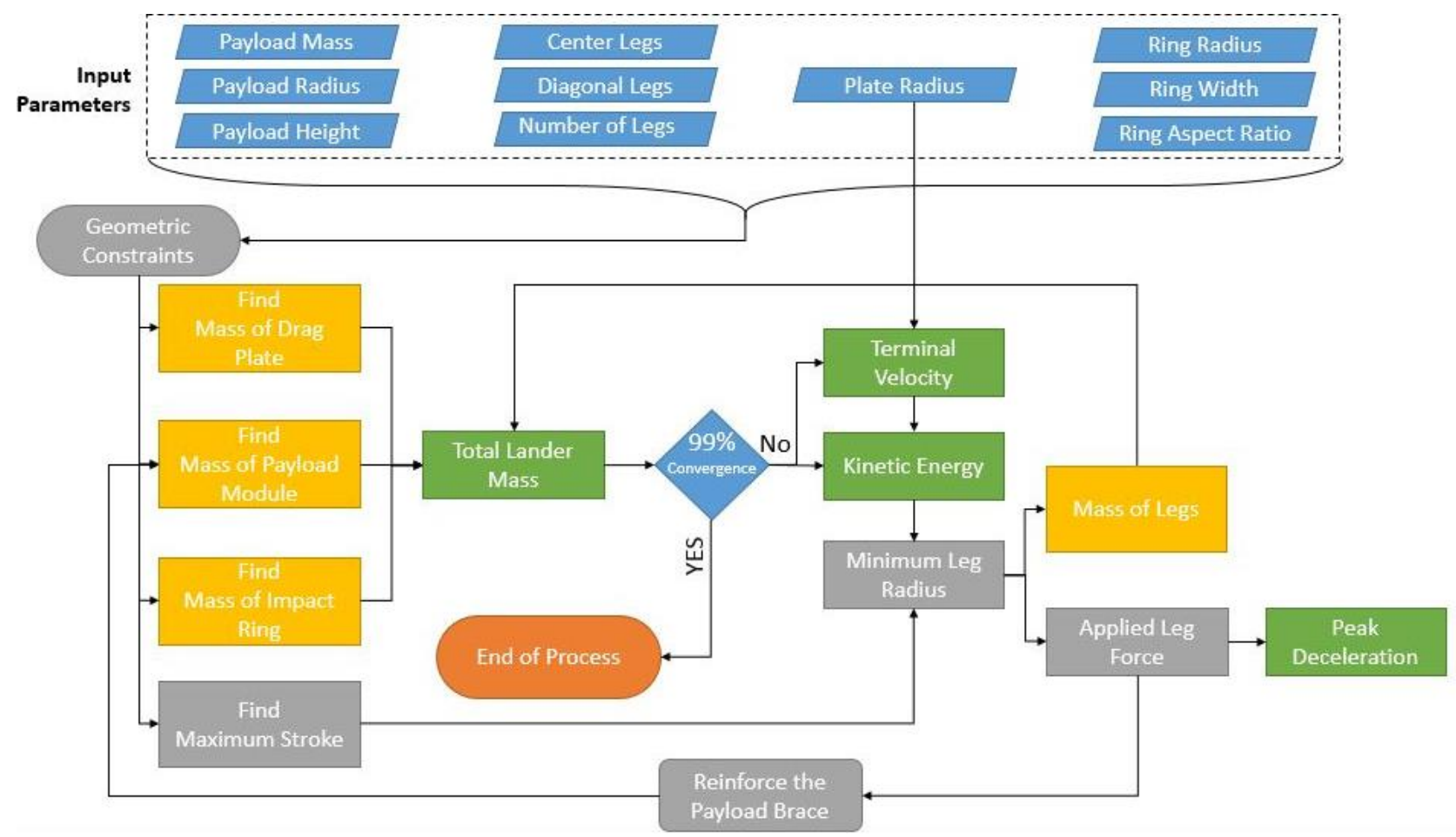

Figure 4: Analytical Model Schematic

Drag Plate: A large amount of computational expense could be invested in order to find the coefficient of drag $\left(C_{d}\right)$ of the lander, however, the purpose of this investigation is to rapidly simulate a variety of different lander configurations. While each configuration can potentially yield a slightly different coefficient of drag. The overall geometry will be similar enough to assume a consistent coefficient of drag. V. P. Karyagin et al. preformed a number of physical experiment on the Venera lander at high Reynolds Numbers (order of 6E6) to find the most accurate prediction of the coefficient of drag would be 0.8 or greater ${ }^{9}$. With the $C_{d}$ known, the terminal velocity was calculated and used to find the total kinetic energy of the lander before impact. 
Payload Module: As mentioned previously, the payload module is comprised of multiple sub-components. These subcomponents include all the scientific payload, the pressure vessel, an internal brace to provide additional stability where the leg connect to the module, and the required insulation. Due to the mechanical focus of this study, only the structural components were modeled explicitly. The non-structural components (e.g., the scientific payload and the insulation) are taken into account by the input parameter (payload mass).

The pressure vessel required two parameters to fully define its geometry, the radius and thickness of the pressure vessel wall. Numerous analytic and experimental investigations of buckling in thin walled spheres under external pressure have been made. Zoelly was the first to provide an analytical solution for the critical applied load given the material properties, radius, and thickness of the sphere and has since been reported in various other works ${ }^{10}$. This equation was used to scale the thickness of the pressure vessel based on the desired radius. Alternatively, an additional parameter can be include to add a factor of safety to the pressure vessel as seen in Eq. 1

$$
t=\sqrt{\frac{F \cdot S \cdot P^{2}}{1.2 \cdot E}}
$$

Inside the pressure vessel where the legs connect to the payload module, there is a brace to add extra stiffness and resist buckling of the pressure vessel. The dimensions of the brace are scaled based on the load applied on the pressure vessel by the legs and the circumference of the pressure vessel at the connection location of the legs.

Legs: The mass of each leg is found from the mass of the honeycomb in the legs, the mass of the leg casing, and the estimated mass of the pin connection joint located at both ends of each leg. To ensure that the lander is able to safely land on the surface given its momentum the amount of energy absorbing material is scaled based on the total kinetic energy of the lander before touchdown. For the analytical solver it is assumed that $90 \%$ of the kinetic energy will need to be absorbed by the honeycomb and metallic foam. Thus the radius of each leg can be calculated such that

$$
r_{\text {leg }}=\sqrt{\frac{\frac{1}{2} m_{\text {total }} V_{\text {terminal }}^{2}-I E_{\text {Impact Ring }}}{\pi \sigma_{\text {leg }} \sum S}}
$$

Where $\sum S$ refers to the total stroke of all of the legs and can be found from the geometries set up by the 10 input parameters. The leg was given the most variability out of any other component. Not only was the length of the leg and its connection point to the payload module left variable, but the number of legs and their configuration were also allowed to change. A trade study was performed to see how sensitive the legs were to the overall design. The three most success- 
ful leg configurations are shown in section IV.C. As seen in Figure 1, the original Venera lander, utilized a Tri-leg configuration with 18 total legs.

Impact Ring: The impact ring was sized similar to the leg. The amount of metallic foam used was calculated

as

$$
I E_{\text {ring }}=\sigma_{\text {ring }} A_{c, \text { ring }} S
$$

Where the cross-section was derived from the two input parameters corresponding to the impact ring, and the stroke was based on the thickness of the foam. A similar equation can be used to find the energy absorbed by the legs.

Peak Deceleration: The payload must be designed to withstand a certain amount of loading. During takeoff and atmospheric entry the lander will experience an elevated g-load. It is expected that the g's experience during entry will be much higher, at an estimated value of $400 \mathrm{~g}$ 's. To ensure the survival of the scientific equipment, the lander must be able to come to a complete stop without exceeding that maximum g's. To check whether the lander configuration is able to impact the surface without exceeding the g limit, an estimated deceleration calculation was included in the algorithm. Because honeycomb supplies a constant force as it deforms, the deceleration will be relatively constant. The deceleration is calculated by dividing the force balance between the legs and the mass of the upper half of the lander by the combined mass of the payload module, drag plate, and parachute capsule according to equation 4 and as shown in Figure 5 .

$$
a=\frac{F_{g}-\sum \sigma_{\text {leg }} A_{c, \text { leg }} \cos \theta_{\text {vertical }}}{m}
$$

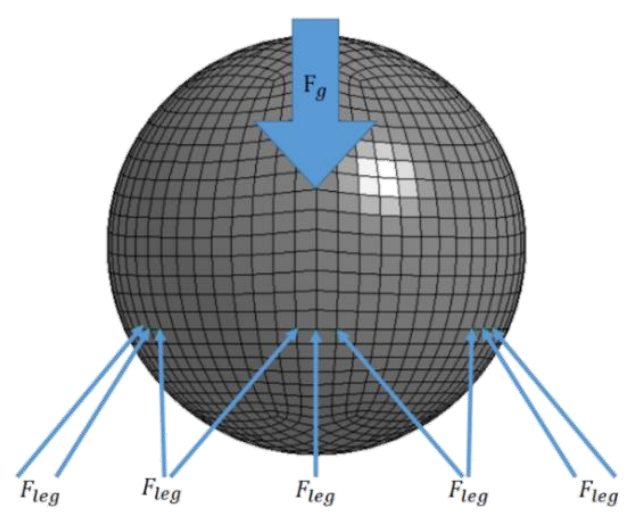

Figure 5: Free Body Diagram for Lander Dynamics 


\section{B. Medium Fidelity Model}

The purpose of this stage of the design tool was to further investigate lander configurations that showed promising results from the low-fidelity analytic solution while retaining a large variability and low calculation time. This model was able to import the calculated masses and initial conditions from the analytical solution and dynamically model them. This allowed for additional parameters to be investigated while verifying the results found by the analytic solution.

In order to reduce calculation time, the lander was modeled using low degree-of-freedom elements such as beams and shells. The pressure vessel is modeled as a hollow shell with an assigned thickness calculated in the analytical solution. The mass of the payload module is evenly distributed throughout the pressure vessel. Because an exact solution was used for the axial loads of 1-D elements, each legs was modeled using a single truss elements. As seen in Eq. 2, the deformation of the legs, and thus the energy absorbed, can be modeled using only the cross-sectional area and the area averaged stress of the honeycomb. A piecewise constitutive relationship, similar to the trend shown in Figure 2, was developed to approximate the honeycomb deformation while the leg radius and approximate leg mass was imported from the low-fidelity model.

As can be seen in Figure 6, the connection points between the legs and the payload module experience a local stress concentration. This is a result of the legs being modeled as 1-D element and each leg was connected to the payload with a single node. Because of this, the stresses observed in the region surrounding the connection point does not accurately predict the actual stress seen on impact. Yet, the simulation accurately predicted the payload dynamics upon impact. To be able to accurately investigate such unexpected stress concentrations, a higher fidelity model was created.

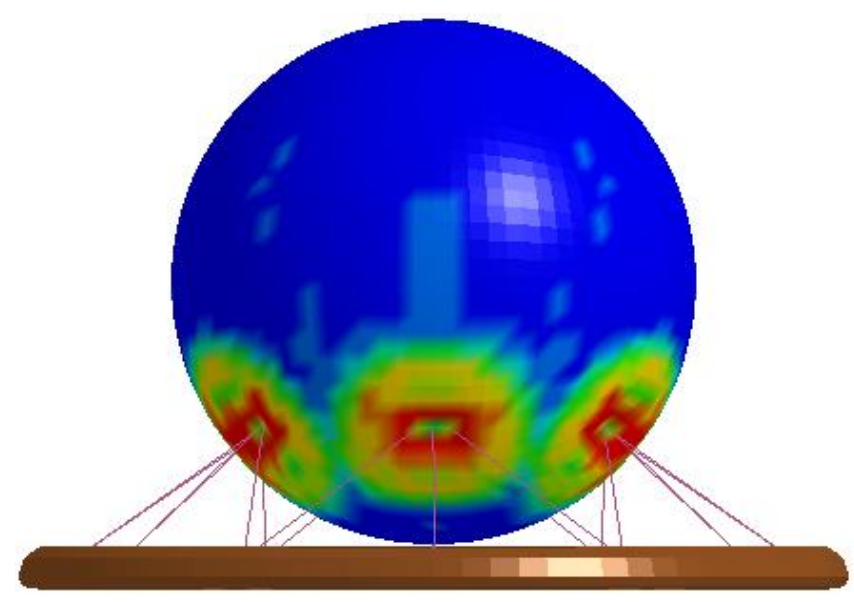

Figure 6: Unexpected Stress Concentration 


\section{High Fidelity Model}

The lower fidelity models were designed to be able to rapidly analyze design variations. In order to achieve short runtimes, the lower fidelity models ignore some of the details of the lander. Unlike the low-fidelity model, the high fidelity model uses 3-D legs and explicitly models the connecting joints so these critical points can be investigated. Additional sub-components, such as the pressure vessel internal stiffener and impact ring frame were captured in both the medium and high fidelity models. As a result of these additional details the high fidelity model yields reasonable stress concentrations around the leg joints. This revealed that for most landing configurations, the stress concentration from the legs onto the payload was benign, however in some cases the initial leg joints were not robust enough to handle loads on impact causing them to fail. In these cases the leg joints were thickened and the simulation rerun. Unlike the medium fidelity model, the high fidelity model was made manually for each design. So each redesign took significant time to prepare.

Once a preliminary high fidelity model was created, a mesh convergence study was performed. The payload acceleration was plotted for three different mesh resolutions with approximate element sizes of $2.2 \mathrm{~cm}$ (coarse), $1.1 \mathrm{~cm}$ (medium), and $0.6 \mathrm{~cm}$ (fine). As seen in Figure 7 if the mesh is too large the payload will decelerate more abruptly due to honeycomb legs being artificially stiffened due to their coarseness. The finer meshes predict a more constant deceleration especially in the latter half of the impact. The peak g's for the coarse, medium, and fine meshes were 48.57, 45.98, and 45.52 respectfully, with a $5.6 \%$ change from coarse to medium and a $1.0 \%$ change from medium to fine.

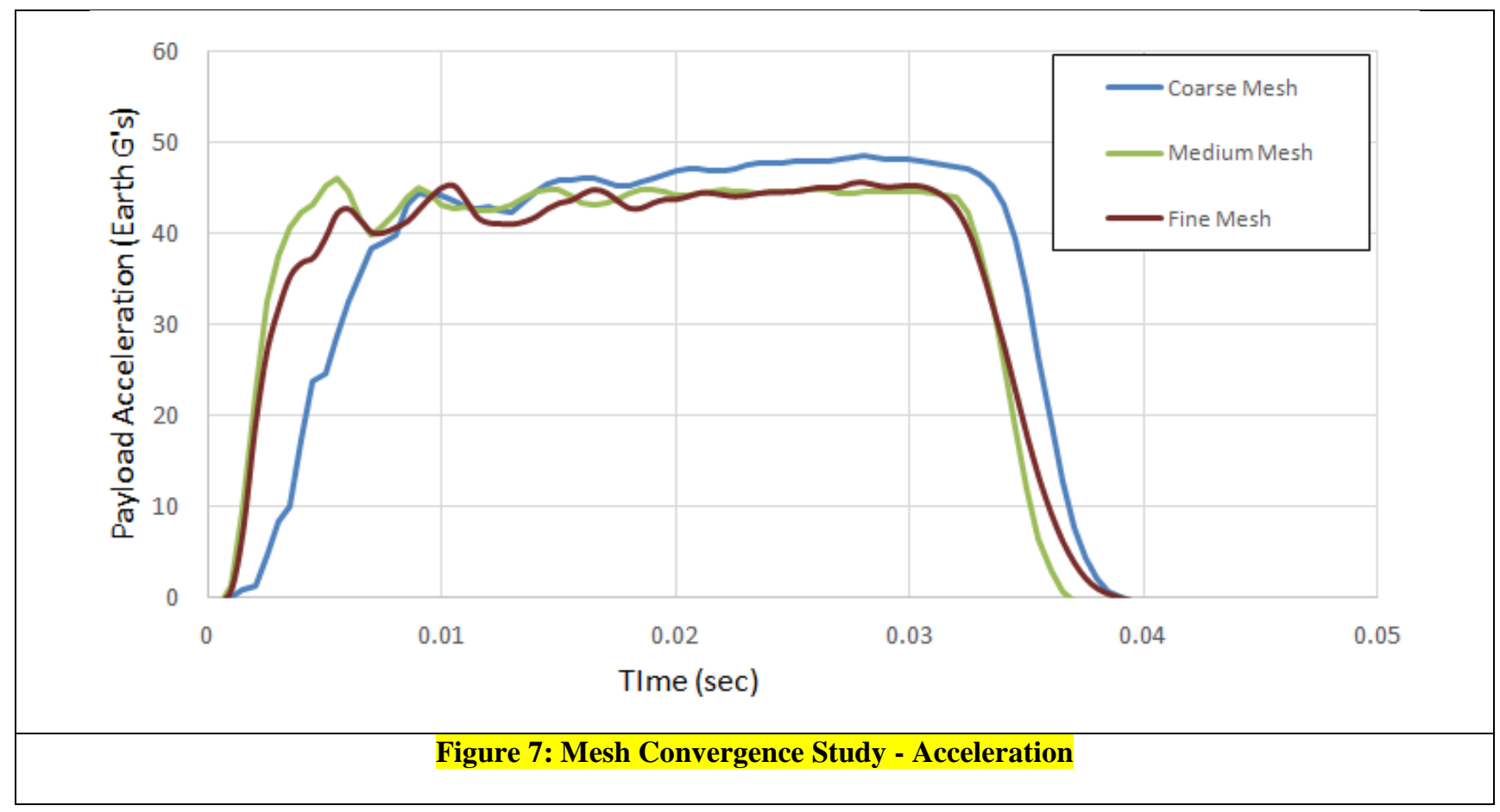


The correlation between the kinetic and internal energies is shown in Figure 8. The internal energy is largely contributed by the plastic deformation of the impact ring and honeycomb legs, while the kinetic energy trend follows the velocity of the payload module. These energies are significant because they quantitatively capture the impact characteristics of the lander. Because a reduced element formulation was used to decrease computational expenses, the total energy was not fully conserved. The change in total energy of the system is $3 \%,-9 \%$, and $-12 \%$ for coarse, medium, and fine meshes respectfully. Typically, simulation that conserver energy well are more reliable. As seen in Figure 8 the coarse mesh simulation generates a significant amount of total energy directly after impact. Although the coarse mesh's initial and final total energies differ by only $3 \%$, the energy generation implies that is simulation is not accurate. The medium and fine meshes loss a little bit of total energy throughout the simulation, but both show a reasonable amount of energy conservation. The energy loses that they experience are largely due to an increase in the hourglass energy, however since there losses are within approximately $10 \%$, they are considered acceptable. Based on the slightly better conservation of energy and due to significantly higher computational cost of the fine mesh, it was decided that the medium mesh size would be sufficient for all further investigation.

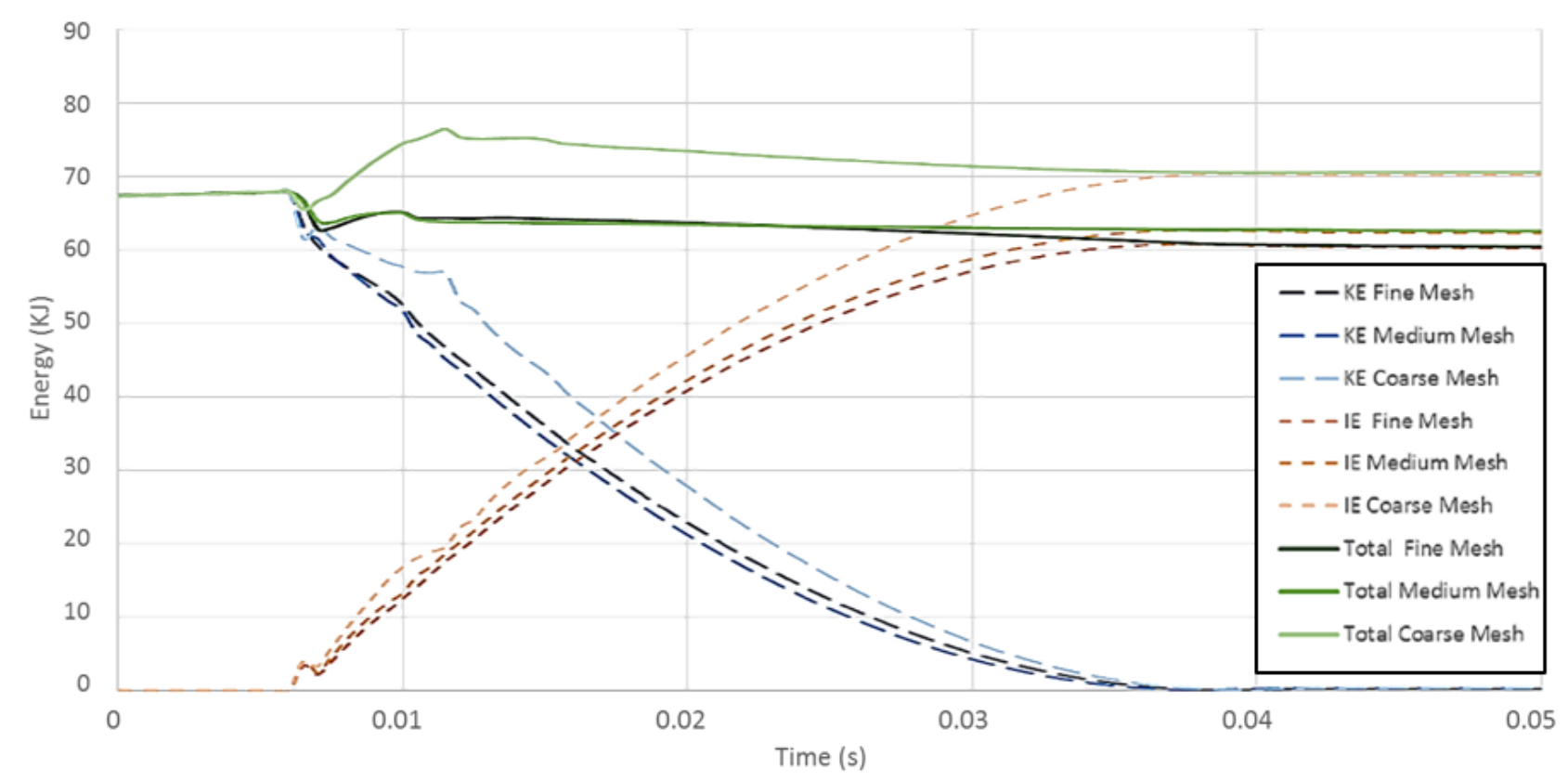

Figure 8: Mesh Convergence Study - Energies

As a result of the detailed modeling of the lander in this model, the unsteady response of the honeycomb under dynamic loading can be observed. The plastic strain predicted in the honeycomb legs is shown in Figure 9. Typically honeycomb crushes axially in a very predictable progressive manner from one end to the other. However as this simulation accurately captures, if the honeycomb crush is not perfectly guided, it will have a tendency to bend back and forth. 
This honeycomb is guided by an outer casing seen in the left half of Figure 9 but the compression is not fully constricted. This is what caused periodic streaks of highly compressed honeycomb. While this does not appear to be a problem for the landing sequence, if it is found to be undesirable, an additional guild rod can be implemented inside each leg to completely constrain this bending.

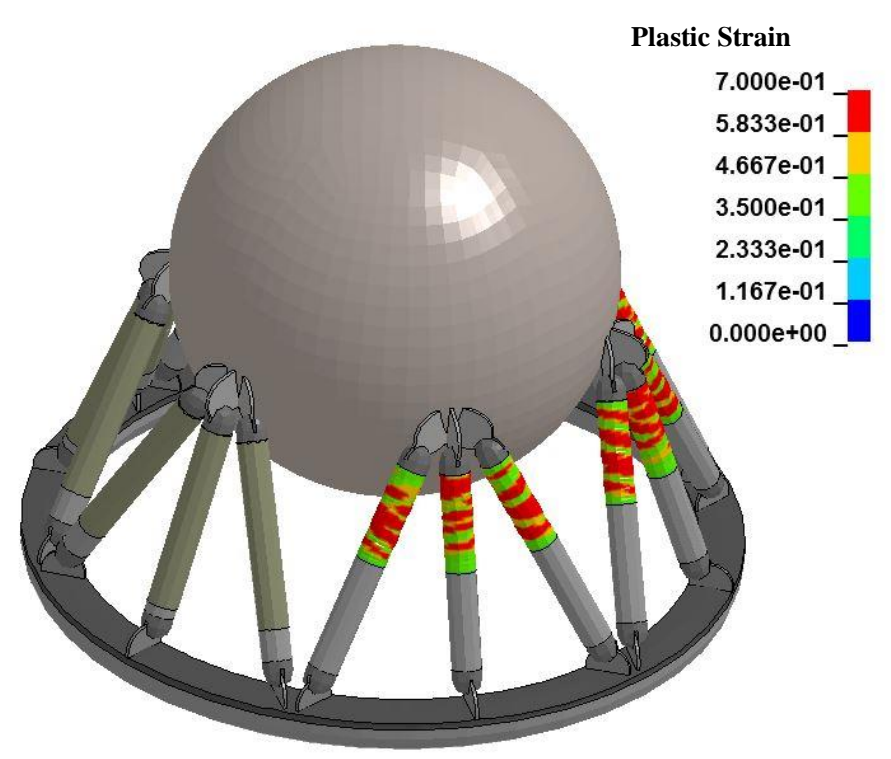

Figure 9: Post Impact Result of High-Fidelity Simulation

\section{Validation}

Comparisons among values taken from all levels of fidelity are shown Figure 10 and Figure 11. By contrasting each of these values, the error in the lower fidelity models can found. The G-loading on impact of a specific design as it was calculated in each of the three models is shown in Figure 10. The energy balance during impact, where the internal energy measures the amount of energy that was absorbed on impact by both the impact ring and the legs is shown in Figure 11. Where the low fidelity values were predicted based on the design and initial conditions of the lander. Keep in mind that the low fidelity model is not a time marching simulation and as such it only predicts maximum values. The time-varying values shown in Figure 11 are there to show the expected trend and were not analytically solved for. Compared to the medium mesh of the high fidelity model, as can be seen in Figure 10 the difference of peak deceleration is $4.8 \%$ and $1 \%$ for the low and medium-fidelity models, respectfully. A $3.1 \%$ and $1.8 \%$ error for the low fidelity model's maximum kinetic and internal energy respectfully, and a $4 \%$ error for both the maximum kinetic and internal energy of the medium fidelity model can be seen in Figure 11. 


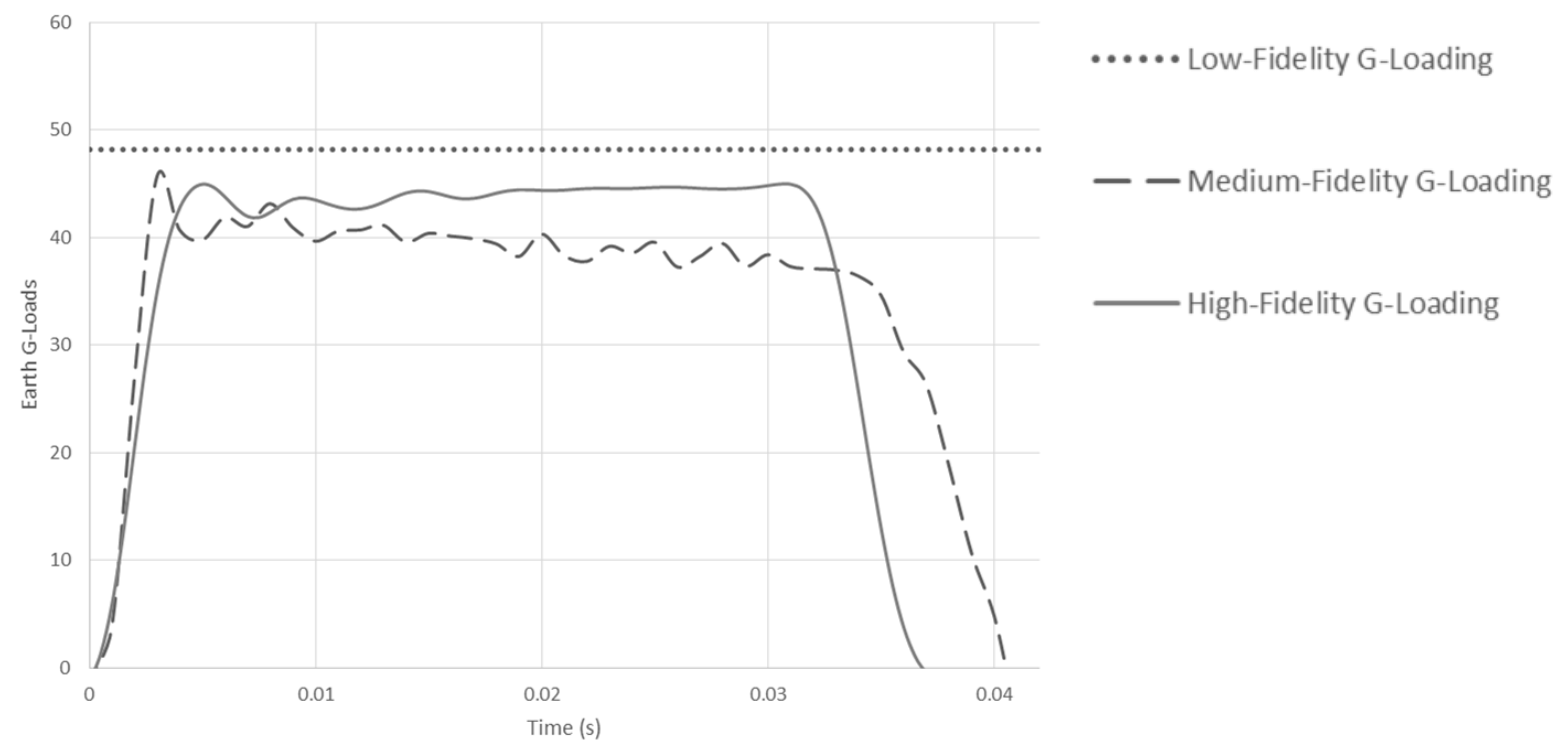

Figure 10: G-Loading Comparison Among All Three Models
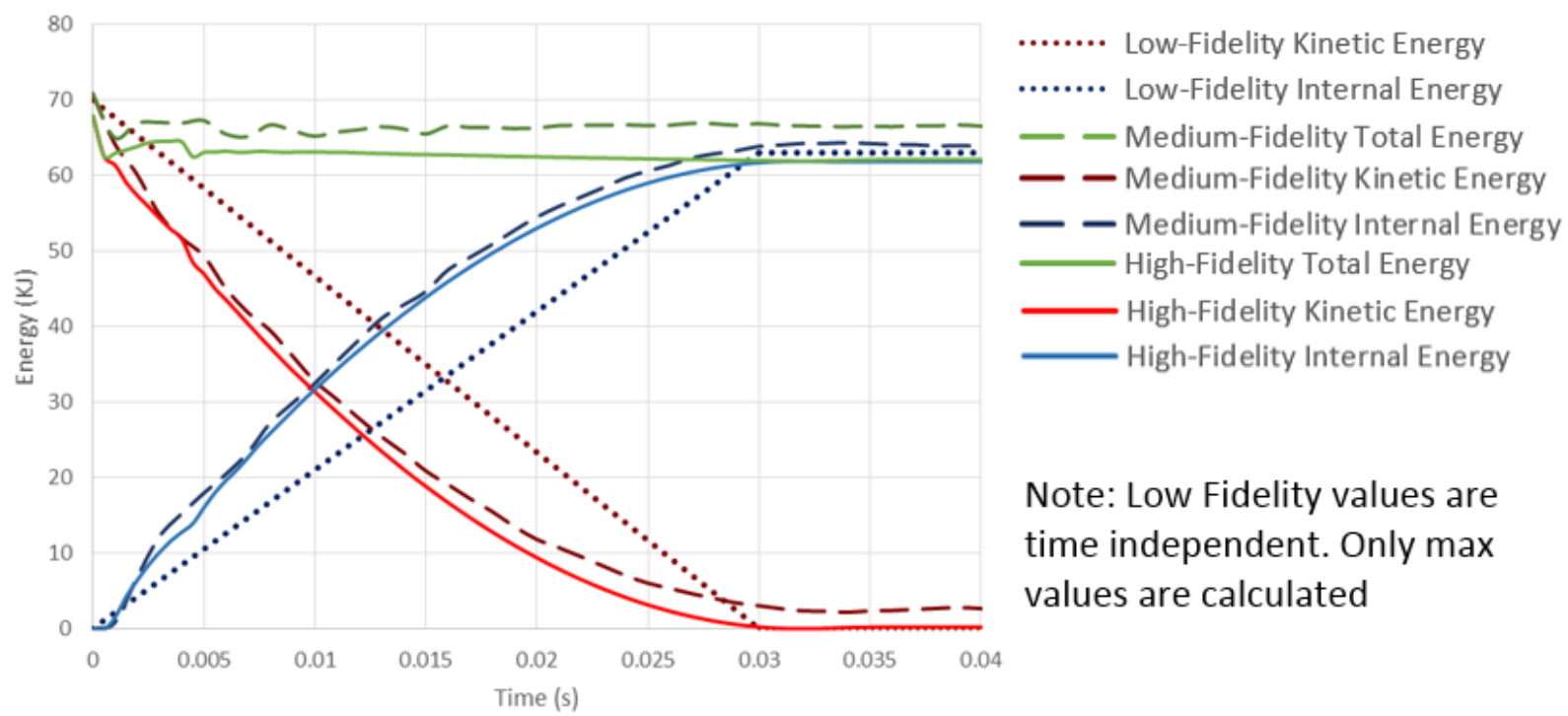

Note: Low Fidelity values are time independent. Only max values are calculated

Figure 11: Energy Comparison among All Three Models

\section{Results - Lander Scalability and Design Sensitivity Analysis}

From the analytical solution, a mass sensitivity analysis was performed. The analyses were broken into three distinct sections: Lander Scalability, Design Sensitivity, and Leg Configuration Analysis. Each of these three sections was used to look at how the change of certain parameters will affect the overall mass of the lander. For this analysis the preliminary baseline design used the validation of the multi-fidelity tool was set as a reference point that all changes could be related to. This preliminary baseline was sized so that its mass would be approximately the same as the Venera 
9 lander. Due to the changes made to the original design the results of the sizing will vary for that of the original Venera landers. However, by comparing the sizing to that of the original Venera, it can be shown that the original design and new design scale similarly and are still comparable.

\section{A. Lander Scalability}

While the fundamental design of the later Venera landers were the same, there were some changes along the way. The Venera 9 and 10 landers were chosen because they closely adhered to the assumptions made in the lower fidelity models. The Venera 9 and 10 landers mass were $660 \mathrm{~kg}$ each. Although the mass of the payload cannot be explicitly found in the literature, based on the sizing algorithm found in this work it was approximated that the lander carried $260 \mathrm{~kg}$ of payload (note that for this work the mass of the thermal protection was included in the payload's mass).

Lander Scalability focused on changing the amount of payload carried by the lander. This is one of the very valuable applications of the analytical solution. As

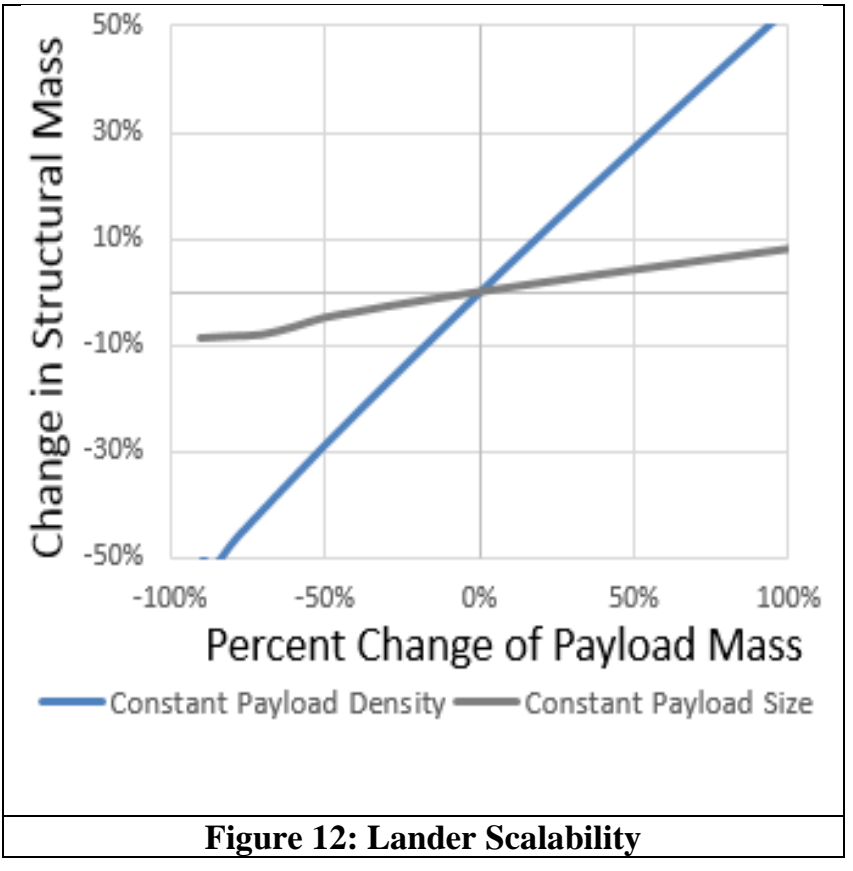
the size and mass of the payload changes, the low-fidelity algorithm scales the lander appropriately. To track these changes, two trends were modeled in Figure 12 and Figure 13: Constant Payload Density and Constant Payload Size. Constant Payload Density shows the change of the lander's structural mass as the size and mass of the payload module scale up and down, while the size to mass ratio of the payload remains constant. This trend line captures the scalability of the lander. The second trend line, titled Constant Payload Size, retains a constant payload module radius

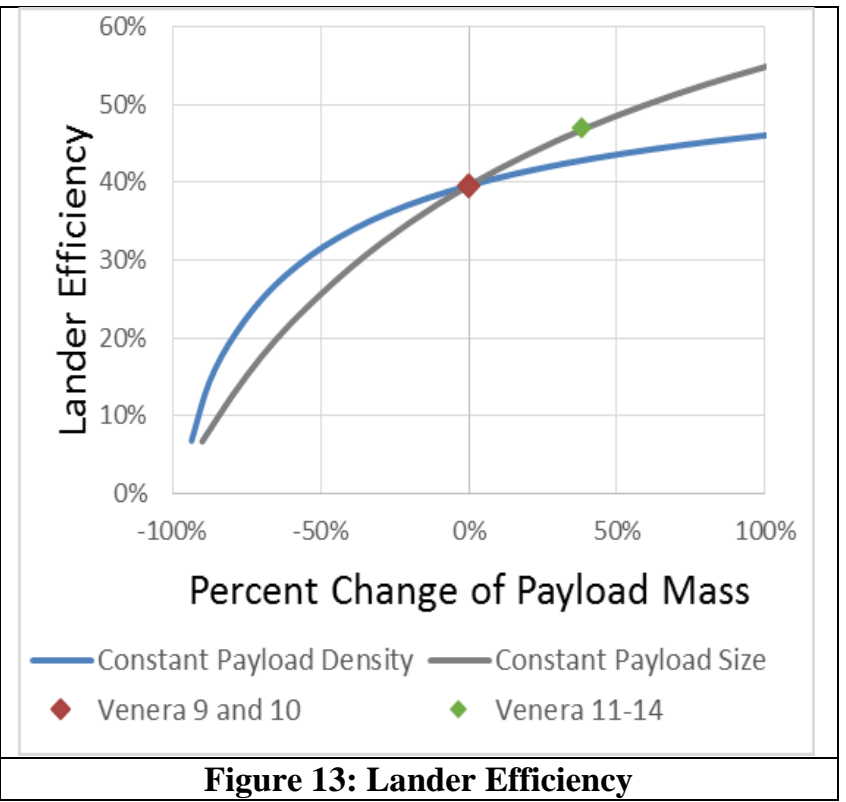
while increasing the amount of payload mass inside the pressure vessel.

As the mass of the payload is increased, the amount of structural support to safely bring it to the surface will also need to increase. This relationship can be seen in Figure 12 and Figure 13. For a constant payload density, it can be 
seen that this lander has a scalability of approximately $2: 1$. That is that if the payload is increased by $2 \%$ the structural mass of the lander will need to be increased by $1 \%$. However, if the payload can be added without changing the size of the payload module the scalability jumps from 2:1 to over 10:1. Venera Landers 11-14 managed to utilize this high scalability ratio by attaching experimental equipment to the outside of the lander. Without making any major changes to the lander design, an additional 100kg of payload was added to the Venera 11-14 landers

The percentage of the lander's overall mass contributed by the payload can be thought of as the efficiency of that lander's design. As the amount of payload increases the efficiency of the lander also increases. This shows that this design scales up well but is less effective for smaller amounts of payloads. As can be seen in Figure 13, the Venera 9 lander had a lander efficiency of approximately $40 \%$. That is, that the mass of the payload made up approximately $40 \%$ of the total mass of the lander. By adding an additional $100 \mathrm{~kg}$ of payload to the Venera 9 lander, the low fidelity model predicted the total mass of the latter Venera landers as $772 \mathrm{~kg}$ which corresponds with an error of only $1.6 \%$.

\section{B. Design Sensitivity}

This analysis was used to reveal the most crucial components of this design. Five of the eight input parameter dealing the structural design of the lander were varied to see the effect they had on the lander as a whole. The remaining three parameters are discussed in the next section. The same preliminary baseline design used in the Scalability Test, was used for the design sensitivity. Each of the parameters were individually varied from $10 \%$ to $250 \%$ of the baseline and the resulting change in the overall mass of the lander was recorded. For example, a 50\% change of most design parameters result in less than a $5 \%$ change in the overall mass of the lander, that $5 \%$ change corresponds to $33 \mathrm{~kg}$. While this value is within the range of uncertainty of the mass approximation, however it is noteworthy and the overall trend will be helpful in selecting an optimize baseline design for future missions.

This analysis is highly nonlinear and many variables are dependent on the others. Therefore, as each component is individually scaled up and down, the other dependent components will also change in size. For example, if the if the payload height is increased the landers stroke will also increase, thus decreasing the radius of each leg and changing the mass of the legs. Because the process is iterative, it is possible that scaling one parameter could affect the mass of every other component. It was found that it was significantly easier and faster to manually search for an optimal design than to program all relevant constraints to find the analytical design sensitivities required for more systematic structural analysis. 


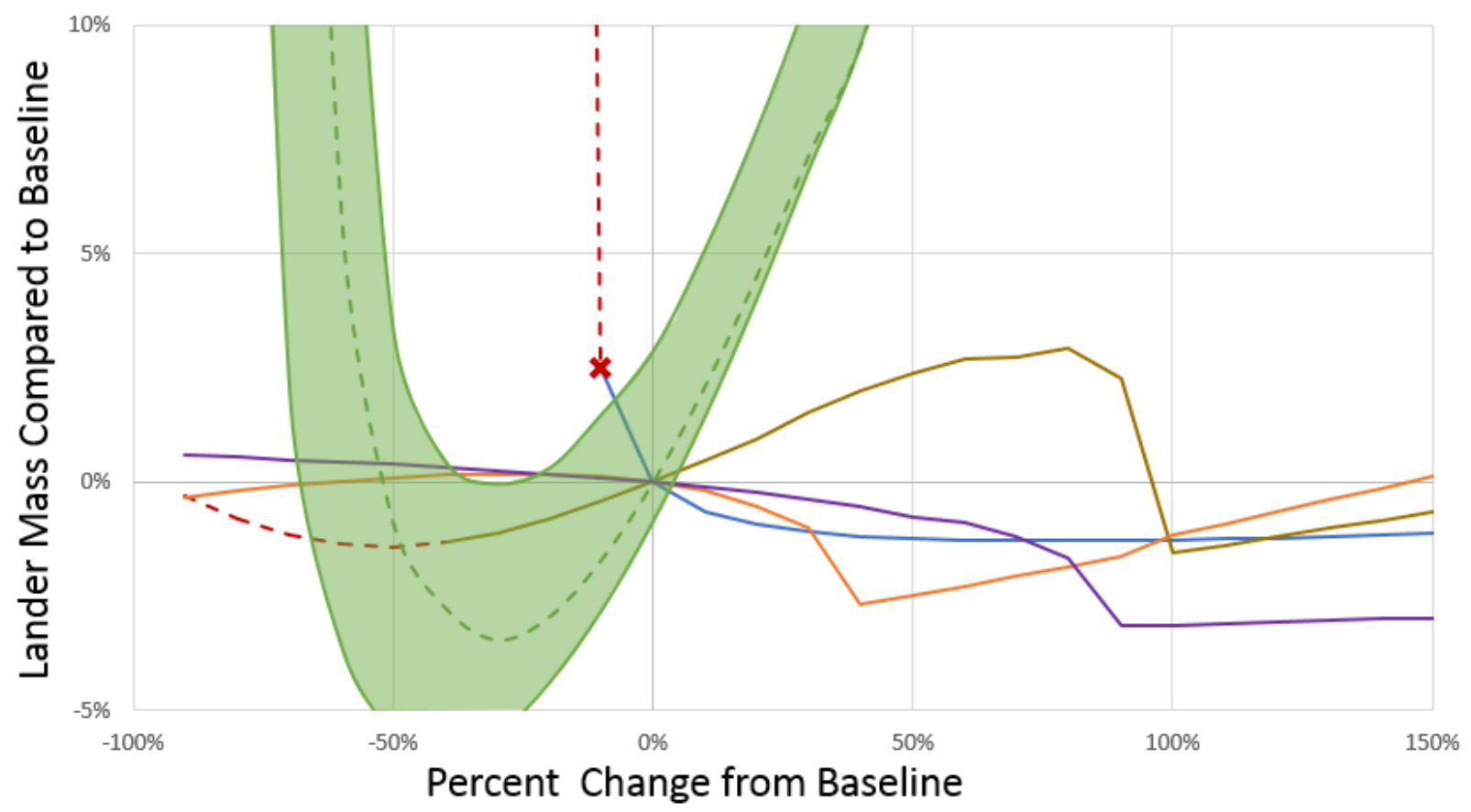

— Payload Height

_- Impact Ring Width

- - - Expected Drag Plate Radius
_ Impact Ring Radius

- Impact Ring Aspect Ratio

Figure 14: Mass Sensitivity Analysis

As seen in Figure 14, the effect the radius of the drag plate has on the mass of the lander was given a range of error. As mentioned previously, the Venera lander had an experimentally found coefficient of drag of at least $0.8^{9}$. Any changes to the geometry of the lander will result in a change the $C_{d}$ of the lander. It was assumed that for most changes the $C_{d}$ would remain close to the baseline value. However because the drag plate has a large effect on the aerodynamic properties of the lander, the $C_{d}$ will change for large changes to the drag plate. As the drag plate radius is scaled down the lander will begin to behave similar to a sphere, although because of the impact ring the $C_{d}$ will never be as low as that of a sphere. As the radius increases the lander will behave more like a flat plate. The $C_{d}$ of both of these shapes at high Reynolds numbers can be found from multiple sources in literature including ${ }^{11,12}$ and can act as the upper and lower bounds $C_{d}$ for Venera-type landers. The expected trend shown in Figure 14 by a dashed line was found by linearly scaling the $C_{d}$ from the lower to upper bounds as the radius varied.

The effect the drag plate has on the lander reveals that there is an optimal value. At some point the mass saved by decreasing the landers speed is overcome by the mass used to extend the drag plate. While future missions to Venus should try to approach this optimal value, it may be prudent not to pick the most optimal point; as the radius is decreased, the impact velocity and g-loading increase. 
The height the payload is above the impact ring, has the least effect on the landers mass of any of the other design parameter. However, if the height is too small the lander's mass skyrockets, because the stroke of legs is decreased, resulting in significantly bulkier legs and a sharp increase in max g-load. After a $10 \%$ decrease in height the lander's g-load exceeded the mission requirement of $400 \mathrm{~g}$ 's, and this is highlighted with a dashed line in Figure 14.

The impact ring's radius, width, and aspect ratio contribute to how much metallic foam is used on the lander. Each of these parameters have a distinct minimum value as they are increased. These minimums reveal a fundamental characteristic of all Venera-type landers. Each of these minimums occur when the impact ring has enough energy absorbing material to fully absorb the landers kinetic energy with no additional support from the legs. At this point, the mass of the honeycomb in the legs is reduced to zero and the legs no longer actuates. As a result, the lander will experience a significant increase in g-load but the expected values will remain well under the mission requirements. This shows that while the leg's offer some practical benefits, like avoiding payload penetration by keep the pressure vessel away from the ground, they do not efficiently assist in the crashworthiness of the lander. As discussed previously, it is a possibility that the original Venera design also did not have any internal energy absorbers although it is not definite. Regardless of the original design, it was decided that the new baseline will not house any internal energy absorbers. Thus a simple solid beam can be used as the design of the legs. Additionally, the size of leg joints can be reduced now that the legs are no longer actuating. Overall this design change simplifies the design and reduces the total lander mass.

\section{Leg Configuration Analysis}

The leg configuration analysis systematically changes the both the number of legs and their configuration. The various leg configuration that were tried in the leg configuration analysis can be seen in Figure 15. The top view of a “normal leg" configuration with 10 legs is illustrated in Figure 15.a. The top view of an "oblique leg" configuration with 20 legs is illustrated in Figure 15.b. And the top view of a "tri leg" configuration with 30 legs was illustrated in Figure 15.c.

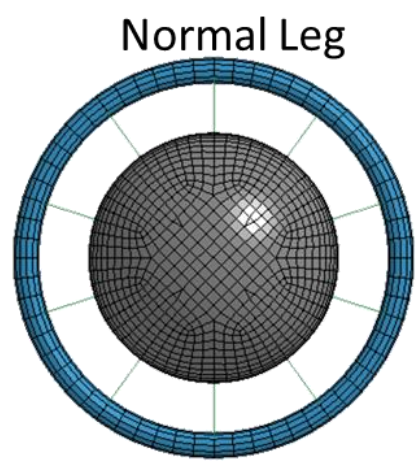

(a)

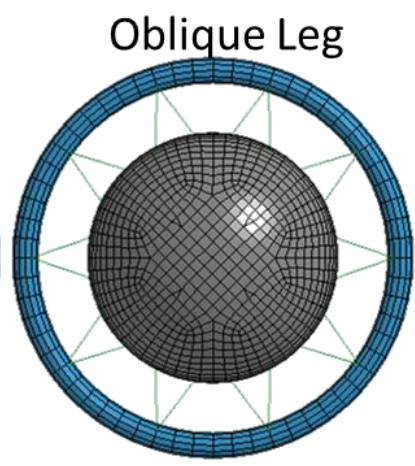

(b)

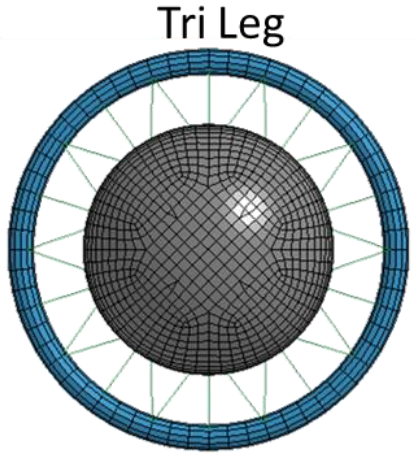

(c)

Figure 15: Various Examples of Leg Configuration 
The leg configuration analysis reveals another significant method to reduce the total mass of the lander. As mentioned previously, the original Venera design had 18 total legs in the tri-leg configuration. As seen in Figure 16, for the same number of legs there is no major change the landers total mass for different leg configurations. On the other hand, the total number of legs have a notable contribution on the landers total mass.

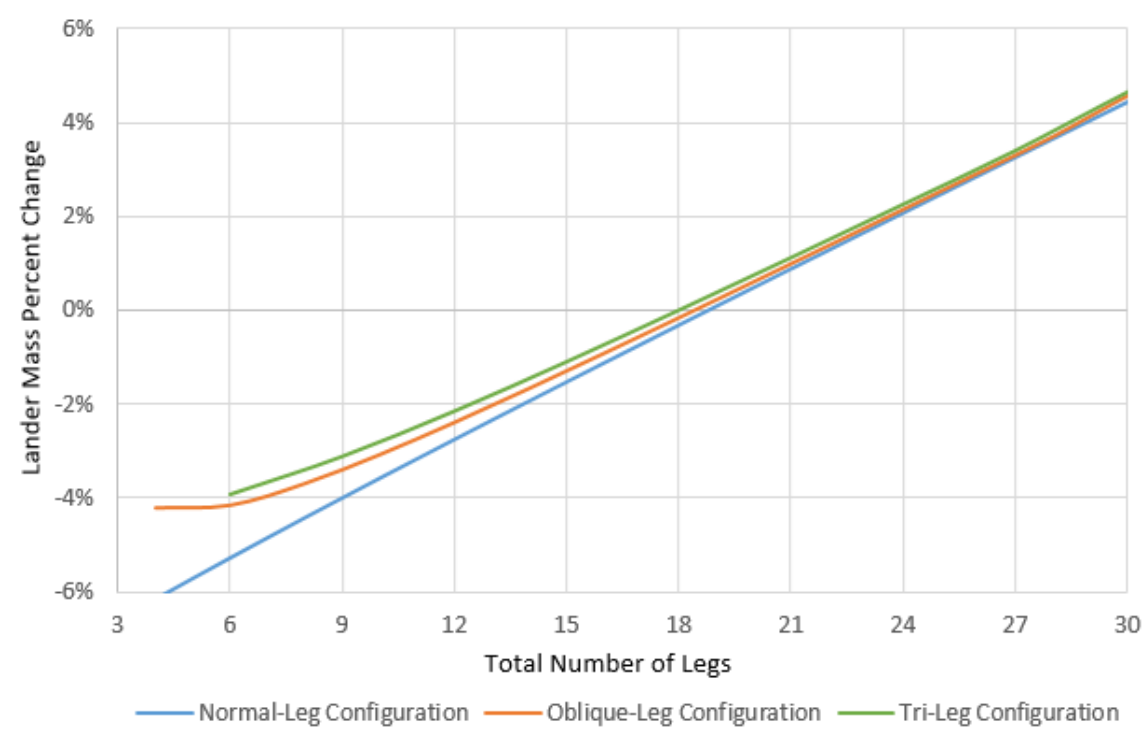

Figure 16: Leg Configuration Analysis

The original design used as many legs as it did to add a level of reliability to the design. If one leg was to fail the effect would not be as devastating as if there were only 6 legs. However, this added robustness comes a cost. As the number of legs are drops, for example in the tri-leg configuration from 18 legs to 9 legs, the total mass savings was approximately $20 \mathrm{~kg}$. Of that $20 \mathrm{~kg}$ more than $15 \mathrm{~kg}$ were contributed to the removal of the hinges and extra fixtures attached to the legs and not the legs themselves. This explains why the effect seen by changing the number of legs so closely matches the Constant Payload Module Size trend seen in Figure 16; most of the leg's mass is just added mass. This conclusion combined with results from the mass sensitivity analysis reveal that the leg are the component with the largest potential for mass savings.

Based on these results the new baseline model for Venera-type landers was chosen. The new design optimizes the lander's components based on a given payload mass of $260 \mathrm{~kg}$. The design variables for the preliminary model and the new baseline model are given in Figure 17. 


\begin{tabular}{|l|c|c|}
\hline & Preliminary Design & New Baseline \\
\hline Payload Height & $0.65 \mathrm{~m}$ & $0.65 \mathrm{~m}$ \\
\hline Impact Ring Radius & $0.9 \mathrm{~m}$ & $0.55 \mathrm{~m}$ \\
\hline Impact Ring Width & $0.025 \mathrm{~m}$ & $0.0625 \mathrm{~m}$ \\
\hline Impact Ring Thickness & $0.050 \mathrm{~m}$ & $0.152 \mathrm{~m}$ \\
\hline Drag Plate Radius & $2.15 \mathrm{~m}$ & $1.43 \mathrm{~m}$ \\
\hline Total Number of Legs & 18 & 8 \\
\hline Configuration & Oblique Leg & Tri Leg \\
\hline Total Mass & $660 \mathrm{~kg}$ & 536 \\
\hline
\end{tabular}

Figure 17: Comparison between Preliminary and New Baseline Model

\section{Conclusion}

A methodology has been created to effectively aid in structural sizing and synthesis of Venera-type landers. Although this work is specifically applied to investigating the design features of Venera-type landers, the methodology can be extrapolated to new designs. Typical investigations of a new lander design require a lot of time to understand how small changes to the design effect the lander as a whole. This multi-fidelity system provides an efficient approach to analysis large variations in a landers design. Thus it can be used to reduce the time and cost associated with investigating a new lander design.

In this work, a purely analytical model was developed which used fundamental equations to size the lander based on the impact conditions. This low-fidelity model proved to be quick and powerful tool for analyzing multiple design variations. It was shown that a design from the low-fidelity can subsequently be modeled in the medium-fidelity level to study different impact orientations and other non-ideal landing configurations. After a design has shown to be very promising from a thorough investigation in the low and medium fidelity models, a high fidelity model can be created that allows the user to better study the joints and leg of the lander.

To validate the analytical model, it was shown that all of the values predicted were estimate within $5 \%$ error of the high fidelity model. After validating the low fidelity model, a mass sensitivity analysis was performed. This analysis was used to track the landers total mass as each parameter was varied. This helps to identify which of the parameters have the biggest impact on the lander mass. An optimal baseline model was designed for a given payload mass of 260 
kg. This work can be extended to any other required payload mass by using the methodology presented in this paper to optimize the lander for any future mission. 


\section{References}

Marov, M. I., and Grinspoon, D. H., The Planet Venus, Yale University Press, 1998.

Bond, P., Exploring the solar system, John Wiley \& Sons, 2012.

Soediono, B., and Kolawa, E., Extreme environment technologies for future space science missions, National Aeronautics and Space Administration, Jet Propulsion Laboratory, California Institute of Technology, 2007. Keldysh, M. V, "Venus exploration with the Venera 9 and Venera 10 spacecraft," Icarus, vol. 30, 1977, pp. $605-625$.

Ball, A., Garry, J., Lorenz, R., and Kerzhanovich, V., Planetary landers and entry probes, Cambridge University Press, 2007.

Masursky, H., Eliason, E., Ford, P. G., McGill, G. E., Pettengill, G. H., Schaber, G. G., Schubert, G., Eliason, E., Ford, P. G., Loriot, G. B., Masursky, H., and McGill, G. E., "Pioneer Venus radar results altimetry and surface properties,” Journal of Geophysical Research: Space Physics (1978-2012), vol. 85, 1980, pp. 82618270.

Doengi, F., Dornier, D. A., Gmbh, S., Sa, A. T., Burnage, S. T., Cottard, H., and Roumeas, R., "Lander shockalleviation techniques," ESA bulletin, vol. 93, 1998, p. 2.

Rogers, W. F., Center, M. S., Hyle, C. T., Foggatt, C. E., and Weber, B. D., Apollo experience report: lunar module landing gear subsystem, National Aeronautics and Space Administration, 1972.

Kariagin, V. P., Kremnev, R. S., Pichkhadze, K. M., Sklovskaia, A. I., and Iaroshevskii, V. A., "Investigation of aerodynamics and dynamics of Venera 9 and Venera 10 during motion in the atmosphere," Cosmic Research, vol. 14, 1977, pp. 741-747.

Zoelly, R., "Ueber ein Knickungsproblem an der Kugelschale,” 1915.

Achenbach, E., "Experiments on the flow past spheres at very high Reynolds numbers," Journal of Fluid Mechanics, vol. 54, 1972, pp. 565-575.

Wieselsberger, C., Further information on the laws of fluid resistance, National Advisory Committee for Aeronautics, 1922. 